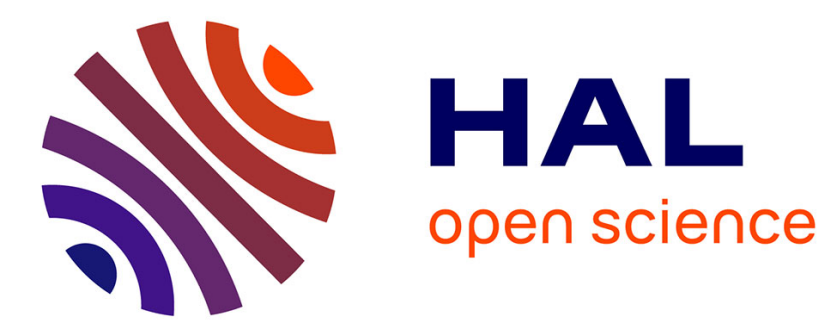

\title{
[INVITED] An overview of the state of art in laser welding simulation
}

Morgan Dal, Remy Fabbro

\section{To cite this version:}

Morgan Dal, Remy Fabbro. [INVITED] An overview of the state of art in laser welding simulation.

Optics and Laser Technology, 2016, 78, pp.2-14. 10.1016/j.optlastec.2015.09.015 . hal-02294144

HAL Id: hal-02294144

https://hal.science/hal-02294144

Submitted on 23 Sep 2019

HAL is a multi-disciplinary open access archive for the deposit and dissemination of scientific research documents, whether they are published or not. The documents may come from teaching and research institutions in France or abroad, or from public or private research centers.
L'archive ouverte pluridisciplinaire HAL, est destinée au dépôt et à la diffusion de documents scientifiques de niveau recherche, publiés ou non, émanant des établissements d'enseignement et de recherche français ou étrangers, des laboratoires publics ou privés. 


\title{
[INVITED] An overview of the state of art in laser welding simulation
}

\author{
M. Dal, R. Fabbro \\ PIMM, ENSAM, 151 Boulevard de l'Hôpital, 75013 Paris, France \\ Keywords: \\ Laser welding \\ Thermo-mechanical simulation \\ Multi-physical simulation
}

\section{A B S T R A C T}

The work presented in this paper deals with the laser welding simulation. Due to the rise of laser pro-cessing in industry, its simulation takes also more and more place. Nevertheless, the physical phenomena occurring are quite complex and, above all, very coupled. Thus, a state of art is necessary to summarize phenomena that have to be considered. Indeed, the electro-magnetic wave interacts with the material surface, heating the piece until the fusion and the vaporization. The vaporization induces a recoil pressure and deforms the liquid/vapor interface creating a vapor capillary. The heat diffused in the material produces thermal dilatation leading to mechanical stress and strain.

As a complete simulation is too large to be computed with one model, the literature is composed by two kinds of models, the thermo-mechanical simulations and the multi-physical simulations. The first aims to find the mechanical stress and strain due to the welding. The model is usually simplified in order to reduce the simulation size. The second, compute the more accurately the thermal and the velocity fields. In that case authors usually search also the size of the weld bead and want to be totally self consistent.

In this review, the major part of equations and assumptions needed to simulate laser welding are shown. Their effects on simulation results are illustrated for each simulation type. The paper aims to give sufficient knowledge and tools to allow a simulation of laser welding.

\section{Introduction}

The laser welding is an increasing process in the whole international industry, for micro-welding as well as deep welding. As other welding processes (with fusion), the assembly of two pieces is obtained by a local fusion and solidification, forming the "welding joint" or "weld bead". The particularity of laser welding is based on the way used to deposit the thermal energy to the piece surface. If with other processes, the heat is transmitted to the piece mainly by conduction and convection (in arc welding, for example). The principle here is to heat the material by the interaction of a light radiation (laser) beam with the material. The energy input responds to the well known optical laws, which means a high temporal stability and a flexibility in terms of spatial distribution. Indeed, the beam shape depends directly on the optical path (fiber, lens, mirror, ...). Thus, it is quite easy to set and change the beam concentration, which leads to a large range of laser intensities and allows a quite large weld penetration scale.

Of course, the research in laser technologies remains more newsworthy than ever and lasers are nowadays more and more efficient in terms of time stability, spatial distribution and energy consumption. Technologies used for welding applications were

E-mail address: morgan.dal@ensam.e mainly $\mathrm{CO}_{2}$ lasers and solid state lasers like Nd:YAG or fiber laser. The $\mathrm{CO}_{2}$ technology was the first able to deliver a power higher than $10 \mathrm{~kW}$ but its wavelength $(10.6 \mu \mathrm{m})$ leads to the incapacity to be transported by classical optical fibers, to be focused by lens and tends to be absorbed by the keyhole plasma. Solid state lasers have a more useful wavelength $(1.06 \mu \mathrm{m}$ for $\mathrm{Nd}$ :YAG and $1.03 \mu \mathrm{m}$ for Yb:YAG), for which the silica fibers or lenses are nearly transparent. Nevertheless, a third category of laser is now able to produce a sufficient power for welding applications (between $5 \mathrm{~kW}$ and $15 \mathrm{~kW}$ ): the laser diodes, which also tend to have better optical properties.

As the beam can be shaped quite easily by users, the laser welding can be realized by two main ways: conduction type or keyhole type. The first produces a weakly penetrated weld bead similar to other processes (arc, for instance). The energy is deposited to the component surface and the beam distribution is shaped to produce low power density only able to fuse the material. The second is obtained from a more dense power distribution, which is sufficient to increase locally the material temperature above to the vaporization one. Due to the recoil pressure (vaporization pressure) a narrow keyhole is created. Depending on the laser wavelength, the beam is more or less well deposited inside this cavity. Thus, the energy is distributed in the material thickness leading to a deep weld penetration (several millimeters to centimeters). 
The consequences of this specific welding case are very interesting in the actual industrial context. First of all, the heat generated by laser can be highly localized (several micrometers) and allows the welding of very small components. Conversely, the very high intensity (power density) generates a keyhole and permit the welding of thick materials. The energy given to the metal is highly concentrated producing very large gradient and thus a small volume thermally affected. Thus, the major effects are few weld distortions associate to large mechanical residual strains. Moreover, the high power density allows very fast welding (until a meter per second) producing in many cases hot cracking.

As the computing hardware is more and more efficient, the numerical simulation is growing in all industrial fields. The welding and more particularly laser welding are also concerned by this fast evolution. The first industrial objective is the design and the optimization of manufacturing processes and components. The simulation is also useful to improve the production sequences and to minimize production problems. That is why a huge amount of model tries to calculate the mechanical effects of welding as residual strains and distortions. Nevertheless, a complete (multiphysical) simulation can also help manufacturers to better understand and to master their processes.

The difficulty in laser welding simulation is the "multi-physical" aspect of the process. As said previously, mainly phenomena are coupled and different scale of physics interacts (photon-electron interaction versus piece shape distortions for example). In spite of the computer efficiency rises, the complete simulation, starting from the light propagation until the structural distortions, is currently not conceivable. For the moment, numerical codes are constrained to simulate the process scale by scale and to concatenate results. For example, the first step is the laser-material interaction (multiple reflection) which gives the heat input to a heat transfer and fluid flow calculation. Then, having the heat distribution with the time, a metallurgical problem can be solved to obtain the material mechanical properties and leading to the mechanical computation. In the literature, multi-physical simulations are not yet really complete simulations, but mainly heat transfer and fluid flow simulations, coupled to a front (or free surface) tracking method and a multiple reflection consideration. Moreover, it should be noted that the difficulty of considering the whole phenomena leads to reduce the contours of the study, this is the case of energy transfer from the laser beam to the material that is mostly approximated by an absorption coefficient.

This state of art tries to give classical tools to choose a model and assumptions for the numerical simulation of laser welding. This paper is built from three main parts. The first is a recall of generalities of laser processes and technologies. The second is an overview of how thermo-mechanical simulations, assumptions and typical results are made. The third concerns the usual steps of a multi-physical simulation, for example the different ways to simulate the beam reflection of the keyhole deformation. At the end a conclusion will be made on the advantages and drawbacks of each method and the potential food for thought on laser processing simulation.

\section{Generalities of laser principle}

In order to introduce laser processing, and before the physical models presentation, a very short presentation of laser principle and technologies is made.

Laser is an acronym for "light amplification by stimulated emission of radiation". As the maser (microwave amplification by stimulated emission of radiation) the laser is based on the stimulated emission founded by Einstein in 1917. The principle is an energy reduction $(-\Delta E)$ of an exited electron by the absorption of an electro-magnetic wave (or photon) whose energy is equal to the electronic transition $\Delta E$. During this energy reduction, an electro-magnetic wave (or photon) is produced and this wave is exactly the same as the first (coherence in space and in time). A laser beam is obtained by accumulating these emissions, between two parallel mirrors. Only photons emitted along the resonator axis are efficient, other directions cannot be amplified. As one of the mirrors has a certain transitivity, the system is open and the beam can be extract from the resonator and used for different applications like welding. As this not the object here, the principle described in this section is very summarized and simplified. Nevertheless, more information and details on the stimulated emission and on the laser effect can be found in the Haken's [28], Siegman's [63] books. In these two references, authors give an overview on laser physics and give basic principles and laws to understand well the laser technologies. While these books are quite old, their instructive facets are very interesting (most recent books written by Svelto [64] or Renk [60]). Weber [72] also gives a quite complete and very accurate review of laser technologies, but focusing on lasing media and on the associated wavelength.

Of course, due to the year of publication (1999), the newest lasers are missing, thin disk laser, for example, which was found also in 1999 [15]. However, most recent books are available $[34,68]$.

The laser beam is a light beam highly monochromatic, thus it is an electromagnetic wave with a spectrum centered at a particular wavelength (depending on the lasing medium) starting from the ultraviolet to the far infrared. As the laser beam is a light, its physics is driven by the classical laws of wave optics that can be found in every book dealing with this subject $[59,19]$. The Dickey's book, written for an engineer or scientist use, gives a broad view of mathematical and physical theory but also many practice examples.

As explained previously, the whole physical phenomena occurring in the workpiece during laser irradiation are highly coupled. While the physic is not easily approachable; the main difficulty remains the numerical computation of this kind of large model. That is why, in the field of welding simulation, some phenomena are commonly brought together and others are uncoupled. For instance, two kinds of model families can be found in the literature: the thermo-mechanical models which aim to compute the effect of welding on the piece mechanical behavior and the so-called "multi-physical" models which try to simulate the process impact on thermal field (weld pool size, thermal gradient ) with as much phenomena as possible.

\section{Thermo-mechanical simulations}

The industry regularly takes part in the thermo-mechanical simulation literature $[45,69,70]$ because the objective is very applied and is about the computation of residual stress and strain induced by laser welding (or other processes). Mainly related to large-sized structures (Fig. 1), this kind of simulation is able to confirm the feasibility of a welding setup. In other words, knowing the material and the process parameters. The model allows to check if residual distortions satisfy the tolerances or if internal strains are coherent with the component use. As the residual distortions are distributed on the whole structure of a component, for very large pieces, numerical methods have been developed but not detailed here, to reduce the size of the numerical problem such as the use of shell elements or local/global approach [21].

As said previously, the simulation of the whole phenomena is not yet possible. Thus, many assumptions have to be made. Firstly, the study contours have to be reduced. That is why the complex interaction of the process with the material is usually reduced to a 


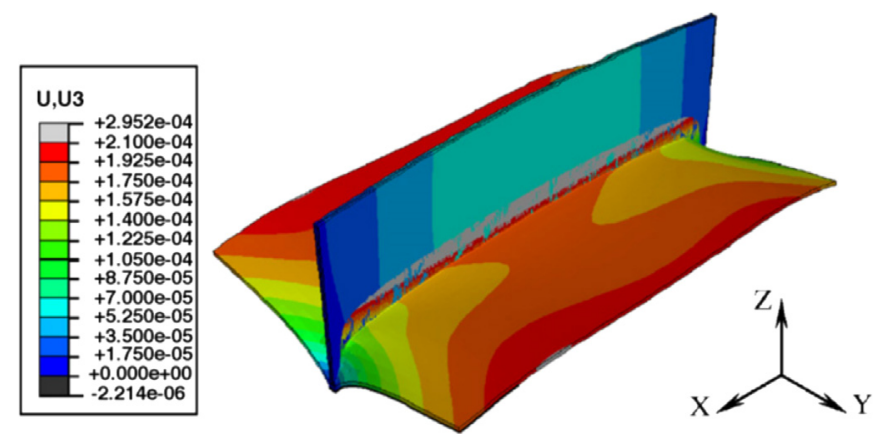

Fig. 1. Example displacement results (pictures from [70]).

volume heat source. The difficulty becomes the setup of this "equivalent" heat source. Indeed, the gas and liquid flows are also neglected and the volume of heat source must reproduce their effects on the thermal field. For instance, the convective effect of the liquid flow, which tends to transport the heat at the sides and at the rear of the fusion zone.

By this way, thermo-mechanical simulations solve only a conductive heat transfer problem and a mechanical problem (Fig. 2).

In the next sections the classically solved equations with the usual assumptions are presented. As the steel is the most welded material, the whole references presented here are about steel or stainless steel welding. Nevertheless, others material properties can be easily found in the literature.

\subsection{Heat transfers}

The heat transfers are the first step of this kind of study because the major consequences of laser welding are due to the thermal expansion.

To compute the thermal field, the classical heat equation (Eq. (1)) has to be solved:

$\rho c_{p} \dot{T}=\vec{\nabla} \cdot \lambda \overrightarrow{\nabla T}+Q_{v o l}$

with $\rho, c_{p}$ and $\lambda$ the thermal properties which are, respectively, the density, the specific heat and the conductivity. It should be noted that, without the liquid flow calculation, the convection term $\vec{U} . \overrightarrow{\nabla T}$ is simplified in this equation.

Here, $Q_{v o l}$ is the volume heat source used to simulate the laser impact on the workpiece. As the keyhole allows the deposition of energy inside the piece, an equivalent heat source defined in the volume makes sense. Nevertheless, finding a satisfactory spatial distribution is not easy. The simplest method is to identify the source distribution from the weld bead shape. Fig. 3 shows a

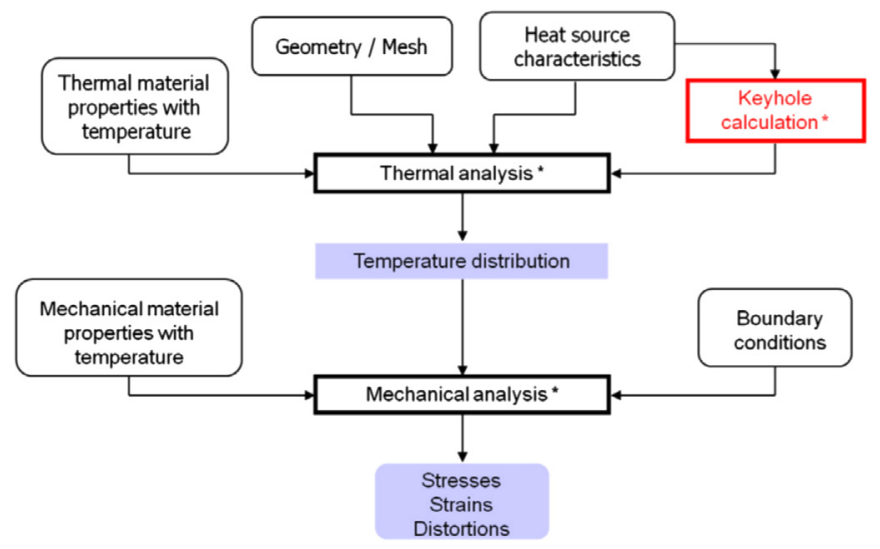

Fig. 2. Example computation algorithm (pictures from [41]).

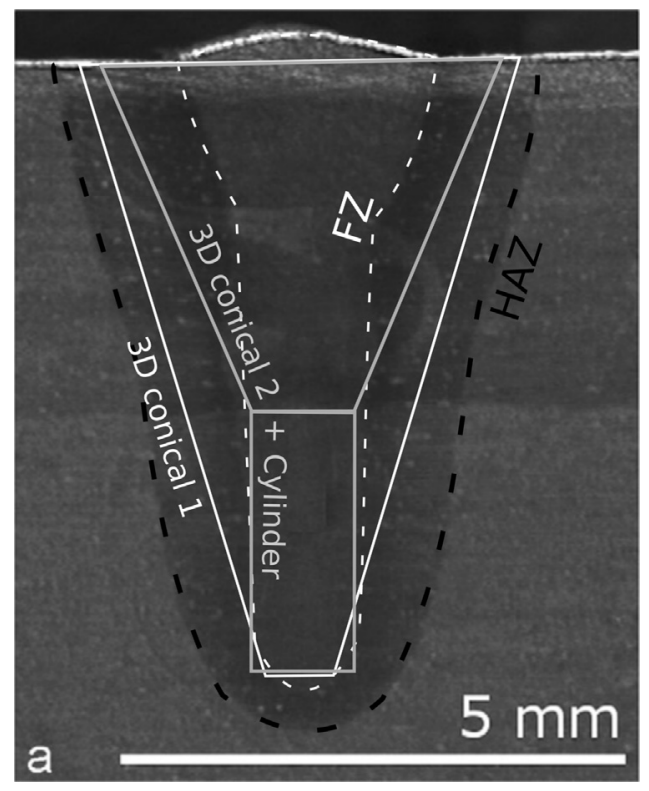

Fig. 3. Scheme of heat source definition (picture from [27]).

macrograph of a laser welding case where the fusion zone (FZ) and the heat affected zone (HAZ) can be seen. In this picture, two examples of volume power distributions have been added. The first "3D conical 1" (Fig. 4a) assumes a fast thermal diffusion and, neglects the FZ shape. The second, “3D conical 2+cylinder" (Fig. 4d) tries to add the particular nail-head shape of the weld pool, which is very classical for laser welding. It should be recalled that in this case, the aim is to obtain a satisfactory temperature field in the solid phase. Thus, a few errors on FZ and HAZ shapes can be allowed. As shown by Rahmann Chukkan et al. [14]), the second source tends to give better results in terms of thermal field.

As the fluid flow is neglected, some phenomena are missing: the thermal convection for example. Two techniques can be observed in the literature to consider that effect:

- The double ellipsoidal heat source (Fig. 4c [14]): Firstly proposed by Goldak et al. [26], this source has a rear part longer than the front one. This particular source "simulates" the effect of the energy transport from the front to the rear of the weld pool by the flow.

- The equivalent thermal conductivity [67] is an arbitrary increase of the heat conduction in the fusion zone. Indeed, this makes sense because the thermal convection increases the heat transfers and this phenomenon can be numerically written as overestimated conductive flux.

Sometimes, a combination of both methods can be observed in the literature [3]. Nevertheless, as these assumptions are parametrized arbitrary, using both of them lead to estimate more parameters (double ellipsoidal parameters and equivalent conductivity).

\subsection{Mechanical stress and strain}

The mechanical computation is here the next step of the calculation. As the number of publications is quite important concerning the mechanical simulation of welding, we will just try to give some references where the mathematical model is well shown (equation and explanations) and where results are interesting.

The solved equation is usually assumed to be the static equilibrium [62]: 
a

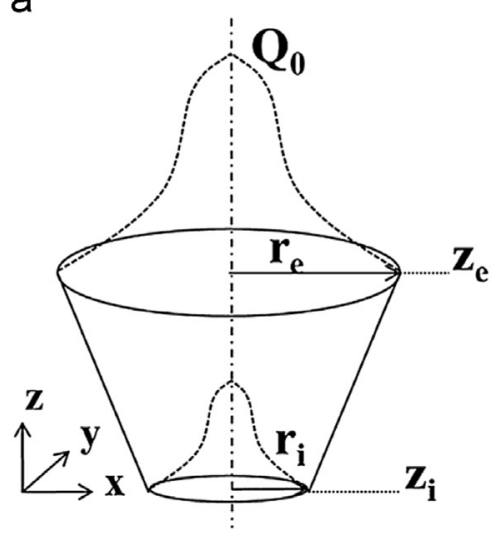

3D conical heat source.

b

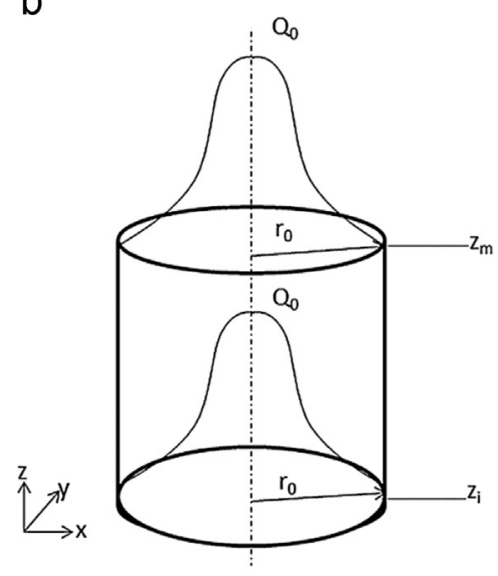

Cylindrical heat source model.
C

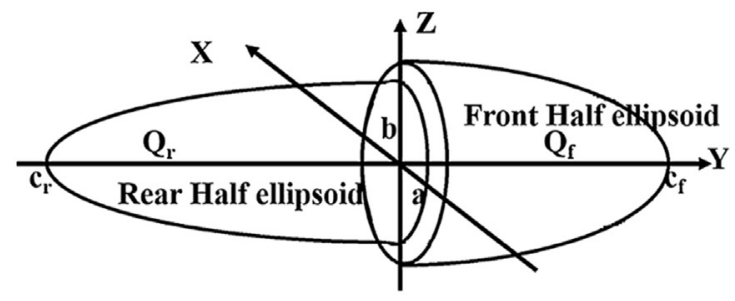

Double ellipsoidal heat source.

d

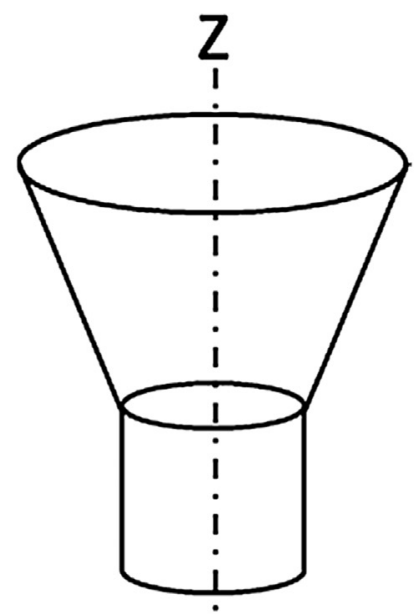

Combination of 3D conical and cylindrical shell heat source model.

Fig. 4. Example of heat source distributions (pictures from [14]).

$\vec{\nabla} \cdot \sigma+\vec{F}=0$

where $\sigma$ is the stress tensor and $F$ is the body forces. The global deformation during welding is defined as the sum of the deformations produced by different phenomena (Eq. (3)). For instance, the elastic strain $\varepsilon^{e}$, the plastic (or viscoplastic) strain $\varepsilon^{p}$ and the thermal strain $\varepsilon^{\text {th }}$ are always considered:

$\varepsilon^{t o t}=\varepsilon^{e}+\varepsilon^{p / v p}+\varepsilon^{t h}+\varepsilon^{t p}$

Depending on the complexity wanted in the simulation, a term considering the transformation plasticity deformation can be added to the previous sum [22]. This phenomenon is a plastic strain due to the volume change observed during a solid transformation, as austenite to martensite for steel. This strain has already been largely studied and they were very well summarized by Fortunier and Bergheau [23]

$\varepsilon^{\text {th }}=\sum_{i} p_{i} \varepsilon_{i}^{\text {th }}(T)$

$\dot{\varepsilon}^{p t}=\frac{3}{2} K S f^{\prime}(p) \dot{p}$

where $p_{i}$ is the phase $\left({ }_{i}\right)$ proportion, $K$ a material parameter depending on the volume variation and $f(p)$ a function of the local phase proportion.

It should be noted that the two lasts strains (Eqs. (4) and (5)) consider the solid phase changes $p$ and its rate $\dot{p}$. Thus, it is necessary to know the behavior of the phase change and one way is to solve a metallurgical problem.

\subsection{Metallurgical model for phase transformation}

The implementation of metallurgical models can have two main objectives: for scientists, the model leads to give accurate information about the phases distribution around the weld bead, for engineers, it is used to determine and finally to control the local mechanical properties of the micro zones of the welds.

Of course, the metallurgical model used for a given simulation depends on the material type. Here, as said previously, only steel behaviors were considered.

Considering reference papers like the one of Kumar et al. [40] well known in the field of welding simulation, it is possible to write the classically used equations:

- The first needed equation corresponds to the ferrite to austenite transformation (during the heating). The Leblond equation [42] is usually used:

$$
\dot{p}(T)=\frac{d p(T)}{d t}=f(\dot{T}) \frac{p_{e q}(T)-p(T)}{\tau(T)}
$$

with $p_{e q}$ being the equilibrium phase fraction, $\tau$ the characteristic time of diffusion transformation which should be different at the heating and at the cooling. But here, the reaction 
a

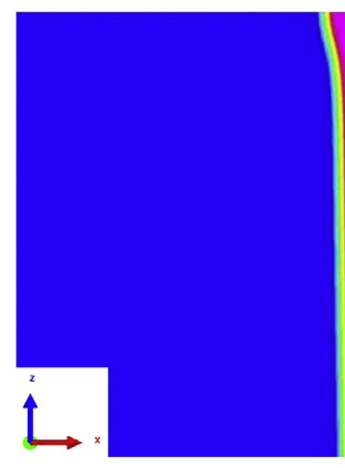

b

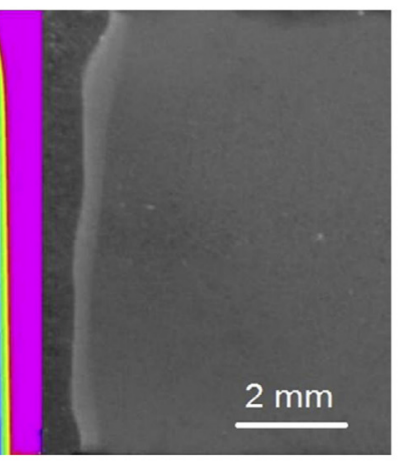

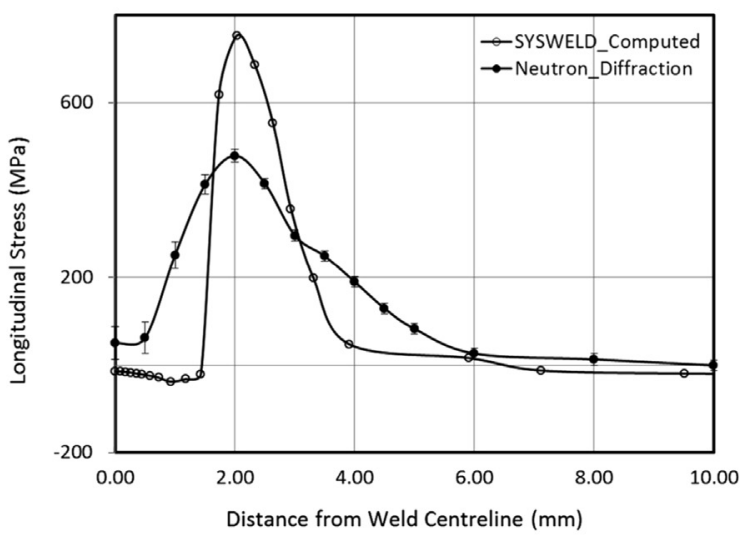

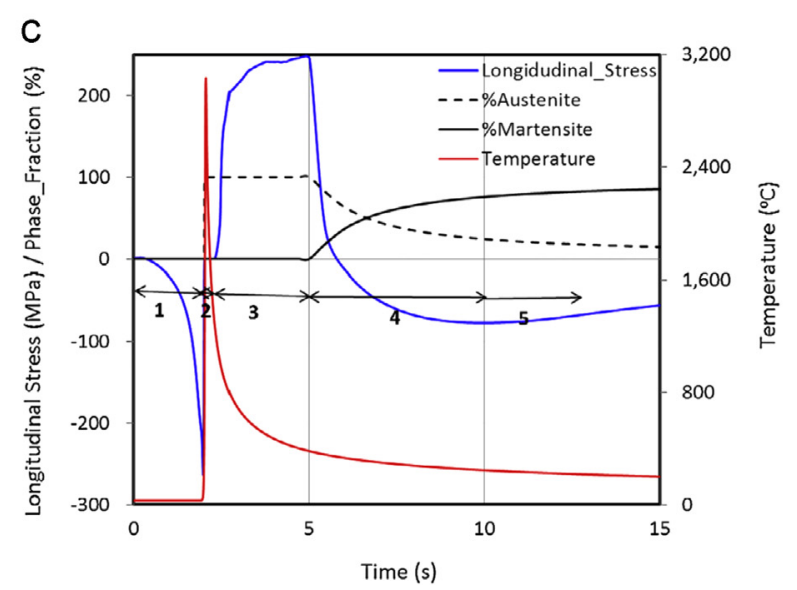

Fig. 5. Simulation results of Kumar et al. [40]: (a) FZ and HAZ comparison, (b) longitudinal stress comparison and (c) phase formation and stress analysis.

occurring during the cooling is a martensite transformation.

- The austenite to martensite transformation is mainly computed by the Koistinen-Marburger equation [38]:

$$
p_{m}(T)=p_{m}\left(1-\exp \left(-b\left(M_{s}-T\right)\right)\right.
$$

For $T \leq M_{S}$ the martensite start $\left(\approx 375^{\circ} \mathrm{C}\right), p_{m}$ the phase fraction at low temperature $(\approx 1)$ and $b$ an evolution coefficient (usually taken to 0.011).

Coupled to a 3D Gaussian double ellipsoidal heat source and with measured mechanical properties, Kumar et al. [39,40] present a quite satisfactory result in terms of fusion zone and heat affected zone (Fig. 5a). They compare the computed longitudinal stress to measurement (neutron diffraction) (Fig. 5b). If the curve shapes and the peak locations are similar in two cases, the numerical one is sharper and the maximal value is largely overestimated. They consider that the error comes from the measurement probe, which is too large and give average values. Finally, they analyze the stress cinematic, showing for example the contraction occurring during the cooling and the phases transitions (austenite and martensite) (Fig. 5c).

This kind of study can be found in many other references [62] or [22]. For example, Ferro et al. [22] have used the same equations (Eqs. (6) and (7)) but they have added the information of austenite grain size computed by the following equation:

$\frac{d D^{a}}{d t}=C \exp \left(-\frac{Q}{R T(t)}\right)-\frac{\dot{p}(t)}{p(t)} D^{a}(t) \quad$ if $\dot{p}(t)>0$ $\frac{d D^{a}}{d t}=C \exp \left(-\frac{Q}{R T(t)}\right)$ if $\dot{p}(t) \leq 0$

where $D$ is the average grain size, $t$ is the time, $C, a$ and $Q$ are positive constants and $R$ is the gas constant. In this study, authors have no experimental validation, but they have analyzed the effects of model simplifications by solving successively models with, all phase transformation effects, only the volume change effect and no phase transition effect. They conclude that the phase transformation plasticity is not negligible.

\subsection{Conclusion}

As shown in the previous sections, the thermo-mechanical simulation of laser welding still highly studied, but the limit of this kind of simulation is the definition of heat source. Indeed, as the thermal field is responsible for the mechanical effects, more accurate studies of the heat transfers have to be done.

\section{Multi-physical simulation}

In this part, the objective is to illustrate the different ways to simulate this laser processing. The present document is split in sections in which each phenomena are described. It should be noted that the major part of references cited here is complete analysis. 
a

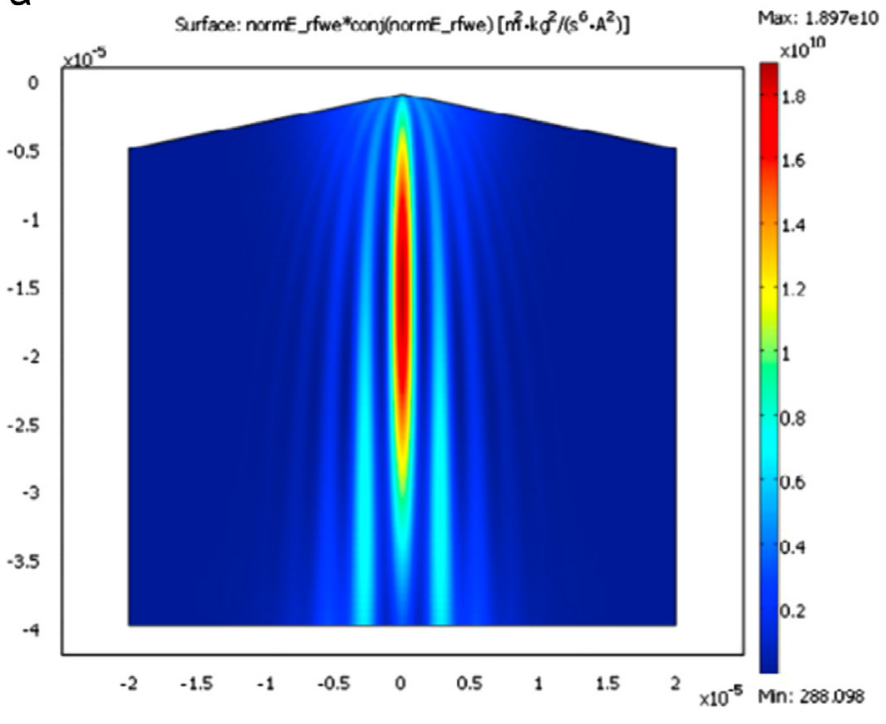

b

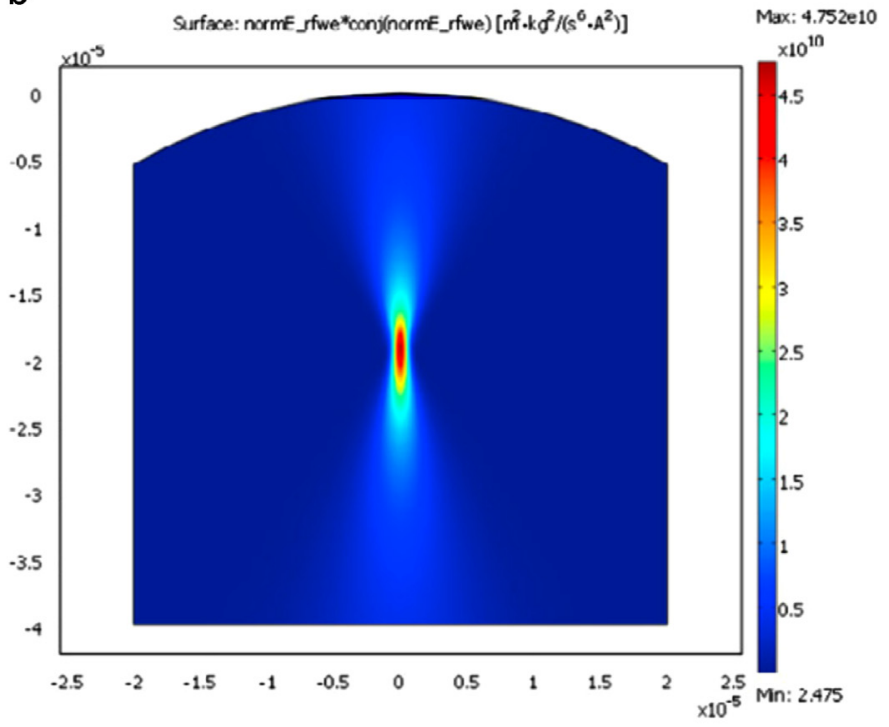

C
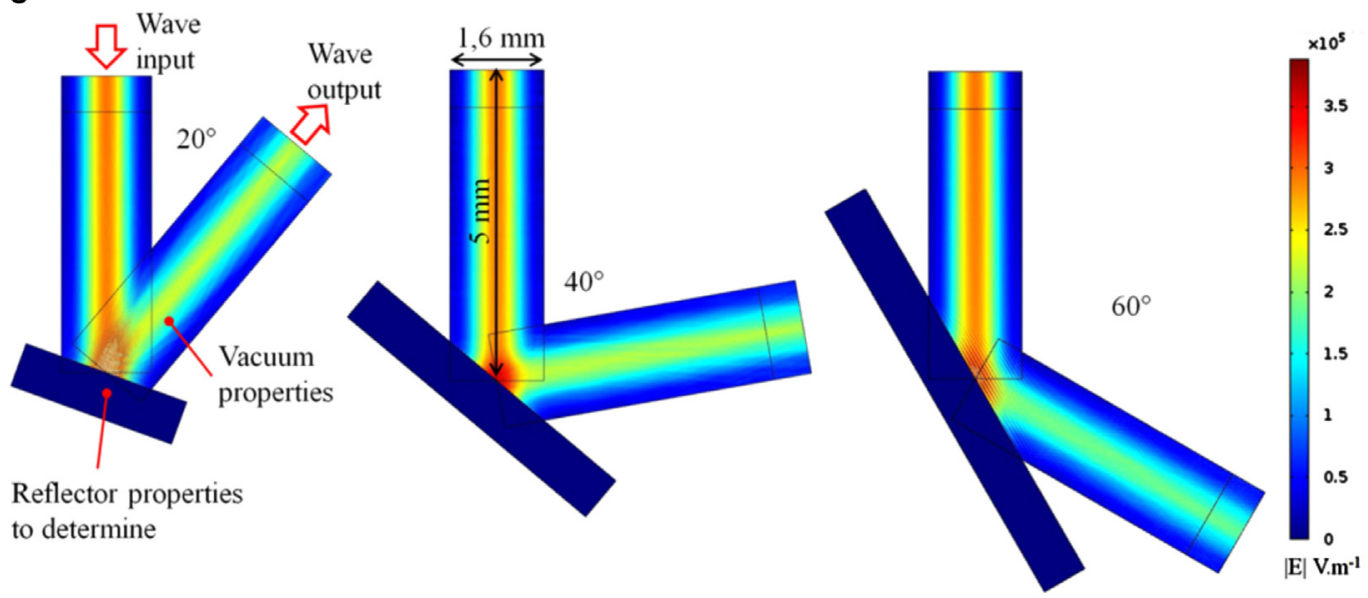

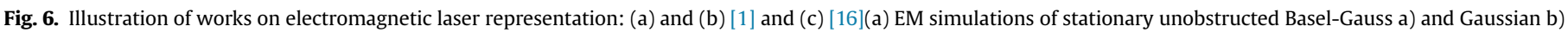
laser beam intensity distributions. (b) model used to determine electromagnetic properties: norm of electric field $\mathrm{E}$ (Vm-1).

\subsection{Laser-material interaction}

The physical interaction between the laser electromagnetic wave and the material takes place at the electronic scale, this is not yet possible to simulate. Nevertheless, as metallic materials are opaques it is easily assumed that the heat transferred from the beam to the metal is localized at the material boundary. The difficulty is the calculation of the beam reflection scheme inside the keyhole and, thus the deposited energy quantity at each reflection. In the literature, two main methods are usually exposed, the electro-magnetic field simulation and the ray tracing method.

\subsubsection{Electro-magnetic simulation}

The principle of this method, is to compute the beam propagation through its electro-magnetic formulation. The solved problem is based on the well known Maxwell's equations. Alexeev et al. [1] have used this kind of analysis to study theoretically the interaction of a pico-second pulsed laser (for texturing applications) with two beam distributions: a classical Gaussian distribution and a Bessel-Gauss beam. They have computed the two beam propagation and effects of different waveguides (Fig. 6a and b)).

Fig. 6 shows also the results of Courtois et al. [16] (Fig. 6c)) which have also simulated the beam propagation for laser welding application. A particular caution has been done on the reflection properties and a validation with classical Fresnel's laws is shown in the paper. Both of them have developed a 2D simulations. Indeed, in this case the mesh (they have used the Finite Element Method) has to be thinner than the beam wavelength ( $\approx 1 \mu \mathrm{m}$ in both cases) and this leads to a very large simulation size. This is a real difficulty because, whereas the models are well defined, an implementation in 3D model is very difficult considering the current computing resources.

\subsubsection{Ray tracing}

As the electro-magnetic formulation leads to numerical difficulties, another model of the beam propagation can be developed, using the ray tracing technique.

The principle of this technique is quite simple and highly used (almost all references [11-13,36,49,52] or [53]). Only the geometrical aspect of the beam is taken. The laser is discretized in "rays" and the objective is to compute, one by one, the rays properties and their interaction with the vapor/liquid interface. As explained by Han et al. [29] two methods can be developed to compute the ray properties: the Direct Search Method based on the computation of the proper discriminant and the Progressive Search Method which needs the resolution of surface equation and ray direction 


\section{collimated beam}

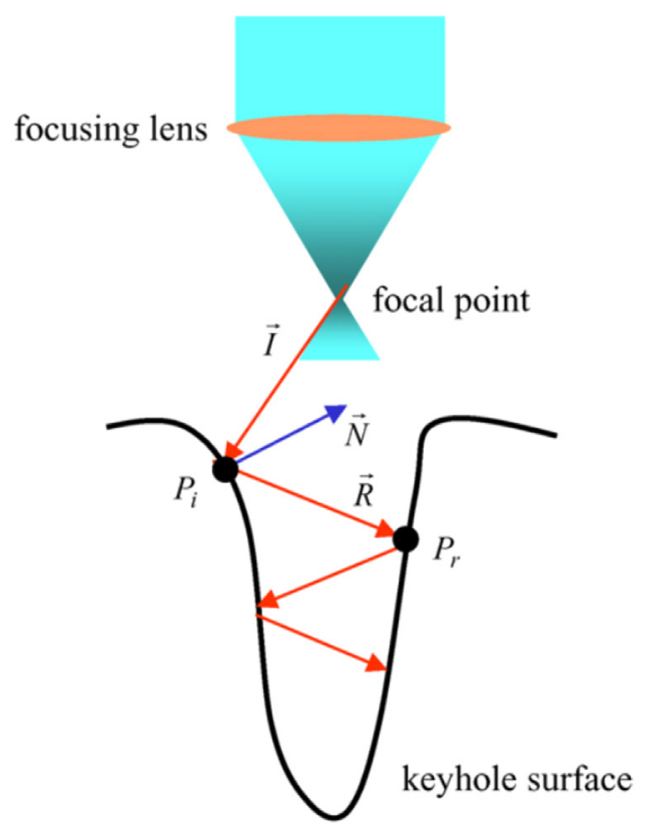

Fig. 7. Illustration of the ray tracing principle [12]shematic diagram of the methodology of multiple reflection effect on the keyhole.

vectors. These two methods give the sequence of reflections properties (Fig. 7) needed to compute the power deposition. At each reflection, the material absorbs a certain intensity quantity (absorptivity) and the remaining "energy" is reflected on an other surface of the keyhole with an angle depending on the refraction index of the plasma. Of course, this process occurs until the complete absorption of the beam or until the ray goes out of the keyhole (Fig. 8).

This numerical step of intensity distribution calculation in the keyhole is highly consuming of computing resources. Both of them are interesting, the electromagnetic formulation allows a realistic description of the beam and the reflection problem is directly solved. The ray tracing method needs the development of a subroutine, but the numerical cost is smaller. It can also be observed that the software or code used for the simulation guides the method choice. This is observed, for example, in [54] where authors have used Comsol Multiphysics, a closed software, but with a lot of coupling possibilities, to solve the electro-magnetic problem and Open-Foam, an open software, to develop the ray tracing method.

\subsection{Heat transfers and fluid flow}

Knowing how the laser power is distributed in the keyhole, the next step is the resolution of the thermal field inside the bulk (solid, liquid and gas phases). Each reference dealing with multiphysical simulation has to treat this part, if the global method is always the same: computing the thermal field and the fluid flow coupled by the convection and the free boundary deformation (recoil pressure and surface tension), some particularities can be observed. In this section, mathematical models and associated assumptions are presented.

\subsubsection{Heat transfers}

The heat transfers are computed by solving the heat equation formulated in terms of temperature (Eq. (10)) or enthalpy (Eq. (11)):

$$
\begin{aligned}
& \rho c_{p}^{e q} \dot{T}+\rho c_{p} \vec{v} \cdot \overrightarrow{\nabla T}=\vec{\nabla} \cdot \lambda \overrightarrow{\nabla T}\left(+Q_{v o l}\right) \\
& \dot{h}+\vec{v} \cdot \overrightarrow{\nabla h}=\vec{\nabla} \cdot \lambda \overrightarrow{\nabla T}\left(+Q_{v o l}\right)
\end{aligned}
$$

The main difference is the consideration of the latent heat of fusion, which is implicit with enthalpy and which have to be added in the specific heat $\left(c_{p}{ }^{e q}\right)$ with temperature formulation [5].

It should be noted that the source term $Q_{\text {vol }}$ is the "volume" laser power and heat losses (vaporization) but it appears only for a particular case, when the free boundary is computed continuously (Level-Set method for example). Indeed, with this numerical technique the front is computed in the bulk, thus it is not possible to set the heat flux as a boundary condition $[36,16]$.

\subsubsection{Fluid flow in liquid}

The fluid flow problem is solved by the computation of two models, the mass conservation (Eq. (12)) and the momentum conservation (Navier-Stokes equation - Eq. (29))):

$$
\vec{\nabla} \cdot \vec{v}=0
$$

$\rho\left(\frac{\partial \vec{v}}{\partial t}+\vec{v} \cdot(\vec{\nabla} \cdot \vec{v})\right)=\vec{\nabla} \cdot\left[-p I+\mu\left(\vec{\nabla} \vec{v}+\vec{\nabla} \vec{v}^{T}\right)\right]+\rho \vec{g}+F_{v o l}$

These equations are written here with the classical assumptions $[13,53,16])$, which means a laminar flow with an incompressible and Newtonian fluid. The solved variables are the velocity field $(\vec{v})$ and the pressure field $(p)$, thanks to material properties, the density $(\rho)$ and the dynamic viscosity $(\mu)$.

In the volume forces term $\left(F_{v o l}\right)$ appear different contributions.
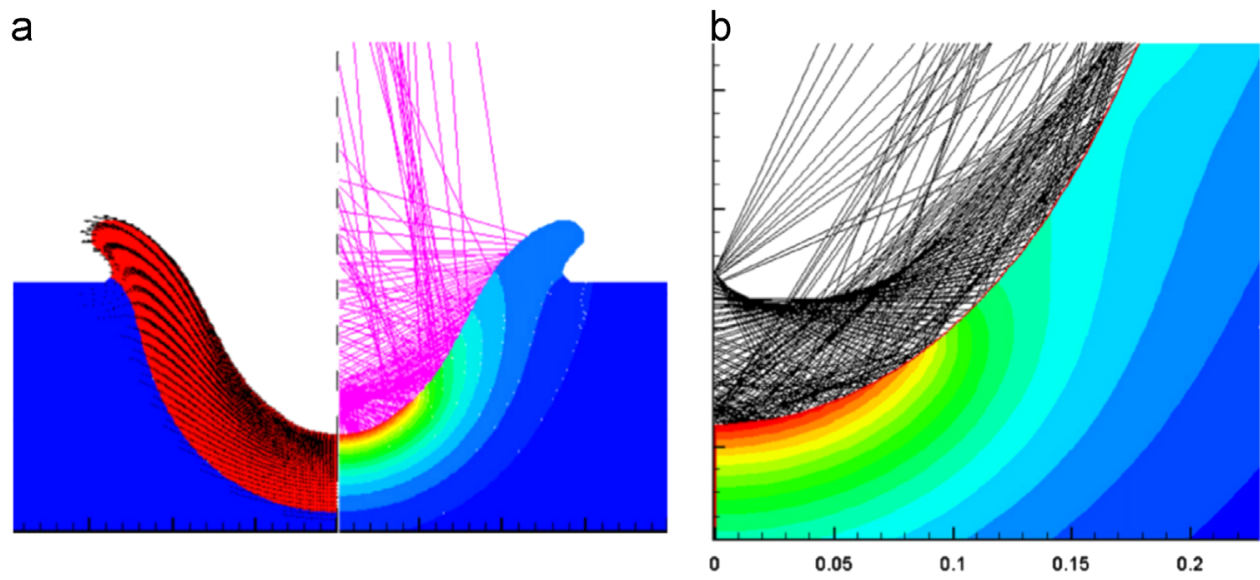

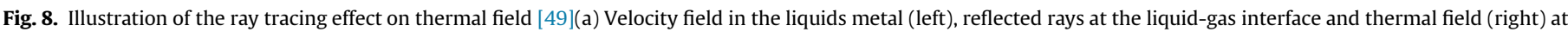
$\mathrm{t}=2 \mathrm{~ms}$. (b) Close-up of the tip of the key-hole: reflected rays at hte liquid-gas interface and theramal field at $\mathrm{t}=7.5 \mathrm{~ms}$. 
a

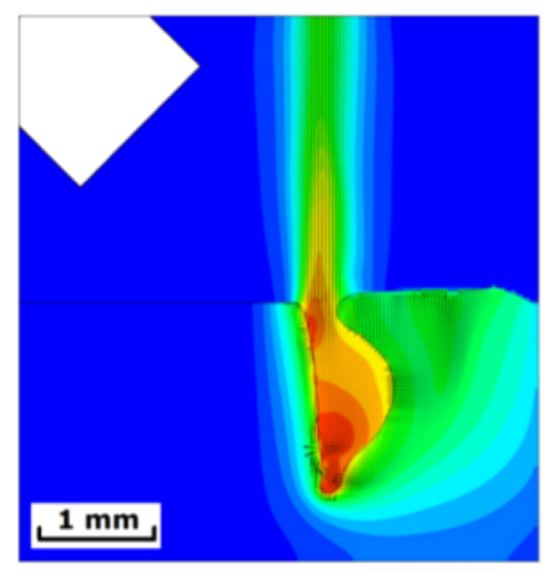

b

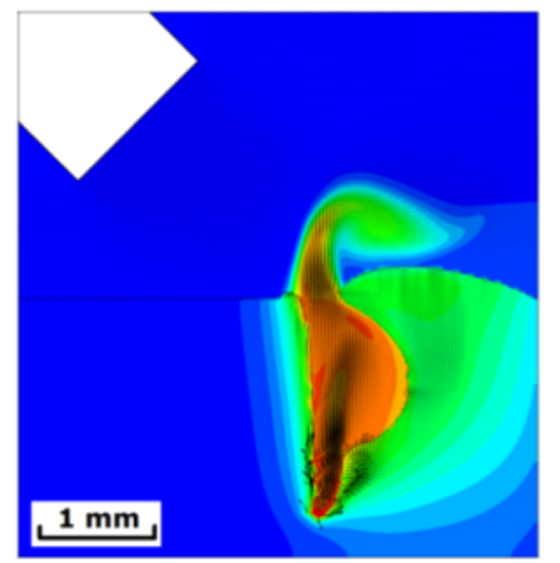

$5 \mathrm{~m} / \mathrm{s}$

(liquid)

$5000 \mathrm{~m} / \mathrm{s}$

(vapor)

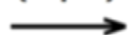

C

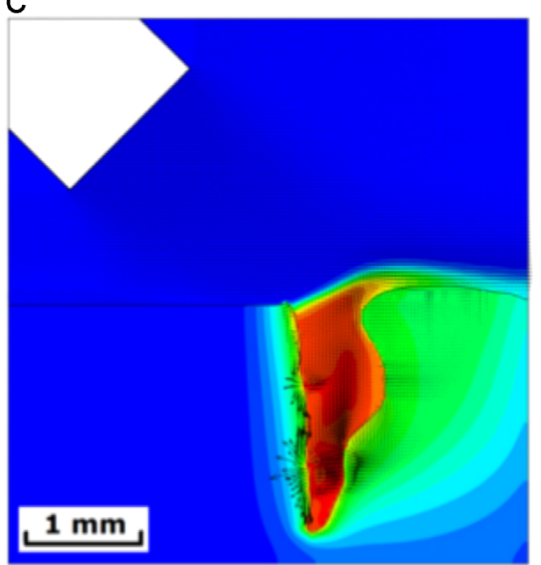

Fig. 9. Vapor temperature field computed by Tan and Shin [66]simulation results on the centre plane for (a) case 5: 0 (b) case 8 : $0.6 \mathrm{CMH}$ and (C) case 9: $1.2 \mathrm{CMH}$.

The most commonly used is the Darcy's condition (or drag force), which associated with the Carman-Kozeny equation [7] allow the description of the mushy zone as a porous medium [6]. The first part of this force is

$\vec{F}_{v_{0 l_{1}}}=-\frac{\mu}{K} \vec{v}$

with

$K=\frac{f_{l}^{3}}{c_{l}\left(1-f_{l}\right)^{2}} \approx \frac{b+f_{l}^{3}}{c_{l}\left(1-f_{l}\right)^{2}}$

Constants $c_{l}$ and $b$ are, respectively, very high (linked to the inverse of inter-dendrite size) and very low (to avoid division by zero in Eq. (14)). $f_{l}$ is the liquid fraction.

Numerically, Eq. (14) is similar a penalization term which tends to zero in the liquid and to the infinite in the solid phase locking the velocity to values close to zero.

An other component appearing in the volume forces is the Boussinesq's approximations used to consider buoyancy inside an incompressible fluid $[13,43,11]$ :

$\vec{F}_{v_{o_{2}}}=\rho \vec{g} \beta\left(T-T_{m}\right)$

This expression is of course activated when the temperature is higher than the molten one $\left(T_{m}\right)$.

It should be added that several studies have been realized considering the effect of turbulence in the molten pool. Chakraborty et al. $[9,8]$ have compared the results obtained by computing the classical laminar Navier-Stokes equation and the high
Reynolds $k-\varepsilon$ model for heterogeneous ( $\mathrm{Ni}-\mathrm{Cu}$ ) pulsed laser welding. They have found very similar results, but the maximal values of velocity and temperature seems to be reduced by the turbulence model. They have also found that the species diffusion computed with turbulence model is more consistent with experimental results.

\subsubsection{Fluid flow in gas}

If the liquid flow is quite well computed, the gas is more difficult due to its compressibility. Sometimes the gas is not simulated [49]. The main advantage of this kind of simulation is to have a real discontinuity between liquid and gas and a smaller numerical problem. Nevertheless, many references consider the gas, but they assume an incompressible gas ([12,18] or [16]). Of course this assumption is false, and the difficulty is to estimate the resulting error amplitude and where it is located. An experimental validation is necessary, but as the keyhole shape is very difficult to observe and to measure precisely. A model validation have been realized by Courtois et al. [17]. They have compared the weld pool temperature and velocity. They have concluded that the error is mainly on the velocity field. That is why, it is necessary to simulate the gas density variation as realized by Otto et al. [53].

In order to take into account the mass variation the previous equation has to be modified:

$\frac{\partial \rho}{\partial t}+\vec{\nabla} \cdot(\rho \vec{v})=0$

Firstly, the density has to be set temperature dependent. In the current stat of art, the more commonly used assumption is to 
consider the mixture of metallic vapor and shielding gas as perfect gas. The density is computed from the classical law: $P V=n R T$. This topic is currently investigated as explained by Otto et al. [53]. At the end of his paper, author explains that is developing a mixture model.

A vapor model has been developed by Tan et al. [65,66]. They have included in their simulation the resolution of the equation of chemical species:

$\frac{\partial\left(\rho C_{i}\right)}{\partial t}+\vec{\nabla} \cdot\left(\rho \vec{v} C_{i}\right)=\vec{\nabla} \cdot\left(D_{i} \rho \vec{\nabla} \cdot C_{i}\right)$

with $C_{i}$ the species concentration (or mass fraction) and $D_{i}$ the diffusion coefficient of an $i$ species. Knowing the vaporized flow rate (Eq. (22)) and with the computation of the vapor concentration, authors are able to consider the laser beam attenuation inside the keyhole and the interaction of the vapor plume with the shielding gas (cross jet). Fig. 9 shows thermal fields computed in the solid, liquid and vapor phases. The figure illustrates the gas interaction over the keyhole.

Authors have also done experiments and the numerical results are in good agreement with macrographs [65].

\subsection{Modeling of liquid/gas interface}

The key zone for laser welding simulation is located at the liquid/gas front. As explained and modeled by Knight [37] the behavior of the keyhole is mainly driven by the thermal cycle of this boundary. Without an excess of details, it is the seat of the laser absorption and of the vaporization process.

\subsubsection{Heat source}

If the laser intensity has a Gaussian distribution (Eq. (20)):

$I(r, z)=\frac{2 P}{\pi \omega(z)^{2}} \exp \left(\frac{2 r^{2}}{\omega^{2}(z)}\right)$

with $\omega(z)$ the standard deviation which depends on the boundary height $(z)$. Due to the multiple reflections, the heat flux transmitted to the metal is thus the accumulation of the absorbed radiation all along the boundary and for each reflection:

$q=\int_{d \Omega}\left(\sum_{\text {reflections }} I(r, z) \alpha\right)$

The latent heat of vaporization (Eq. (21)) is also localized at the free boundary. Its formulation observed in the literature is a Hertz-Langmuir equation modified (Eq. (22)) considering the recondensation parameter $\left(\beta_{r}\right)$ :

$q_{\text {vap }}=\dot{m}_{\text {vap }} L_{v a p}$

$\dot{m}_{v a p}=\sqrt{\frac{m}{2 \pi k_{b}}} \frac{p_{\text {sat }}}{\sqrt{T}}\left(1-\beta_{r}\right)$

with $m$ the atomic weight of metal, $k_{b}$ the Boltzmann constant and $p_{\text {sat }}$ the saturation vapor pressure.

The thermal boundary condition is a combination of the two previous terms:

$-\lambda \vec{\nabla} T=q-q_{\text {vap }}-q_{\text {losses }}$

with $q_{\text {losses }}$ the convective and radiative losses.

\subsubsection{Vaporization pressure and surface tension}

During the phase change, the mass transfer from liquid to vapor, located at the boundary, produces a vaporization pressure. Also, due to the high velocity of gas ejection, a hydrodynamic pressure is produced by the shearing of the vapor over the liquid and finally, the surface tension tends to close the keyhole.

The pressure caused by surface tension is well known (YoungLaplace equation: $\Delta p=\gamma \kappa$ ) and its variation with the temperature (Marangoni effect) can be set, for steels, by the Sahoo's law [61]. The recoil pressure is more difficult to define. For instance, some references [10] are entirely devoted to try to define well this function. Nevertheless, the most recurrent formulation is based on the Clausius-Clapeyron equation:

$p_{\text {sat }}=p_{0} \exp \left(\frac{M L_{v}}{R}\left(\frac{1}{T_{\text {vap }}}-\frac{1}{T}\right)\right)$

Semak and Matsunawa [46] have proposed the relation $P_{\text {rec }}=A p_{\text {sat }}$ with $A$ a numerical parameter equal to 0.55 [4]. Used in many references $[2,25,12]$, this relation can be completed by adding a re-condensation term: $P_{\text {rec }}=\left(1+\beta_{r}\right) p_{\text {sat }} / 2$. With $\beta_{r}$ the retro-diffusion parameter [30], this means the percentage of recondensate vapor. For high evaporation level, $\beta_{r}=0.18$ giving the first recoil pressure expression.

The boundary condition is an imposed pressure:

$p=p_{\text {rec }}+p_{g}-p_{a m b}+\gamma \kappa$

with $p_{a m b}$ the atmospheric pressure far from the keyhole, $p_{g}$ the partial pressure of surrounding gas (near the target surface) different to the atmospheric one, $\gamma$ the surface tension and $\kappa$ the curvature. Forgotten in many references, the ambient pressure term has a strong effect on the evaporation behavior. Neglecting it, the user simulates in fact welding under vacuum conditions and at the very beginning, the keyhole tends to appear before the vaporization temperature [31]. In the literature, authors like Pang et al. [57,58] have treated this effect with particular caution in complete simulations. They have shown that the recoil pressure $p_{\text {rec }}$ is equal to the ambiant one $p_{a m b}$ for a surface temperature smaller than the evaporation temperature corresponding to this ambiant pressure; For temperatures greater than this evaporation temperature, the recoil pressure is then defined by $p_{\text {sat }}\left(1+\beta_{r}\right) / 2$.

\subsection{Modeling of liquid/gas interface}

The previous interface phenomena should be introduced in a model as boundary conditions, in the thermal problem and in the fluid flow problem. Nevertheless, the simulation of the interface liquid/gas can be realized by different ways: Lagrangian methods (ALE) or Eulerian methods (Levels-set or VOF).

\subsubsection{Arbitrary Lagrangian Eulerian (ALE) method}

The Arbitrary Lagrangian Eulerian [32] method is developed to calculate the boundary nodes displacement physically and the bulk nodes arbitrary. This means that the solutions inside the domain are computed with the Eulerian formulation. Thus, the mesh motion is established in order to maximize the mesh quality.

This method was used for example by Medale et al. ([47,49] or [48]) to simulate laser welding and as the interface corresponds to a geometrical boundary of the system (Fig. 10), the sources can be written as shown in the previous section and applied directly at the boundary.

Fig. 10 shows the mesh solutions after two time steps. As the time increases, a mesh constriction is observed (bottom of the keyhole). This numerical result is critical because in finite element method (fem), the solution depends on the mesh quality and in laser welding the interface deformation is significant. A re-meshing process could be interesting to avoid too high distortions. The main advantage of this technique is the well definition of the front discontinuity. Nevertheless, it should also be added that this technique is not adapted at all to "closed" frontiers (bubbles). This 

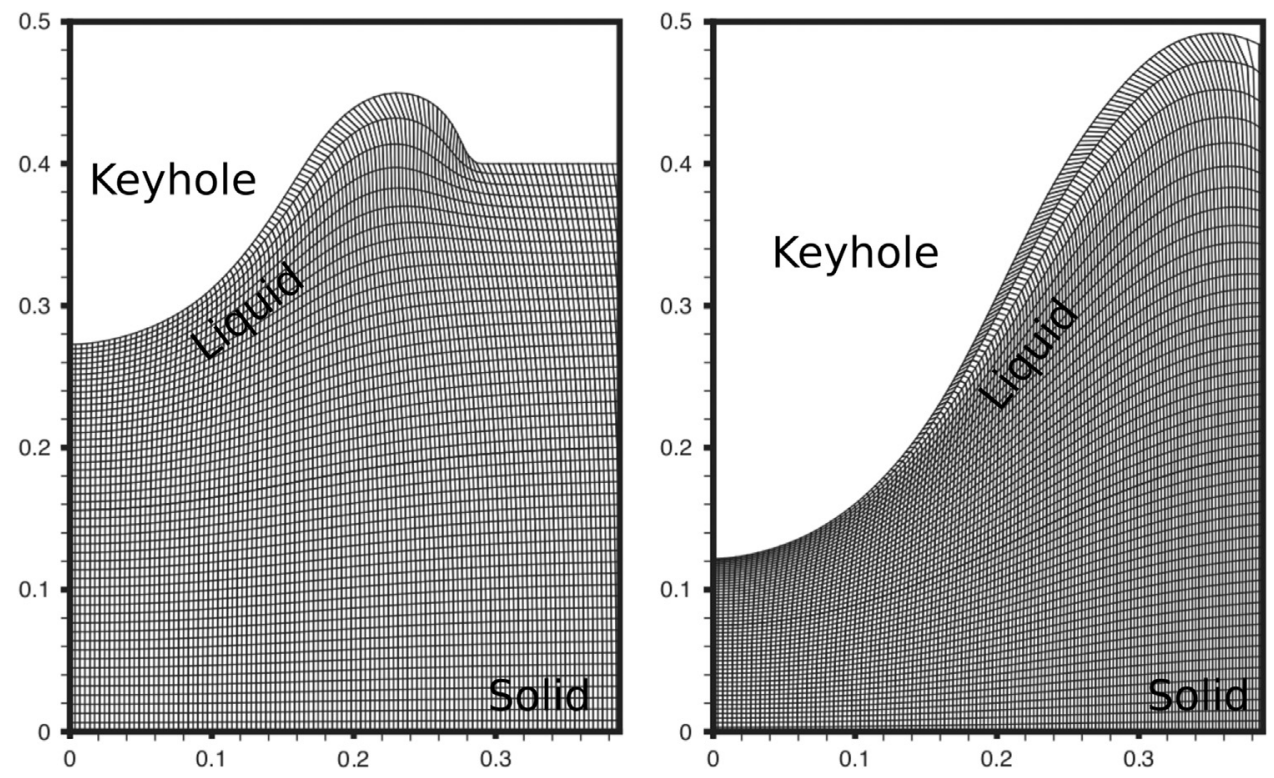

Fig. 10. Illustration of mesh distortions [48].

limitation is highly problematic to simulate laser welding which produces quite often porosity.

\subsubsection{Volume of fluid (VOF) method}

The VOF method [33], mainly associated to the finite volume method (to solve partial differential equations), is a "free surface tracking" technique. Here, the physical equations of the two domains (liquid and gas, for example) have to be in Eulerian formulation (fixed grid). The fluid flow is computed through the mesh and the VOF method is used to localize and treat the free boundary motion. This method is usually described in three steps:

1. Determination of the location and shape of the free boundary.

2. Computation of the front displacement with the time.

3. Application of the boundary condition.
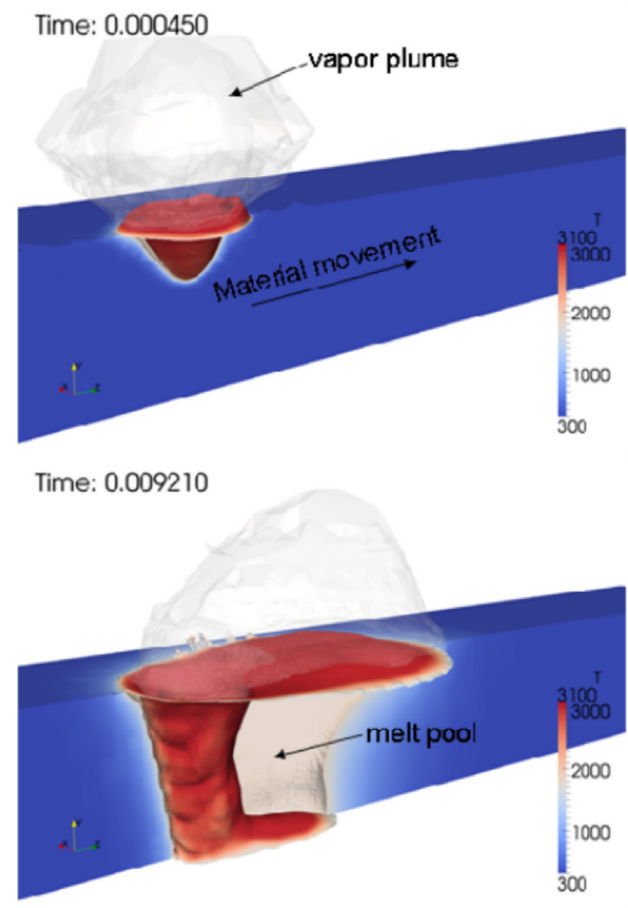

This method is based on the definition of a function $(F)$ defining the fluid state. $F$ is a step function equal to one if a mesh cell is full of fluid and zero if the cell contains no fluid. Between zero and one, $F$ is the volume fraction of fluid and a cell must contains the interface. After having detected the cells containing the front $(0<F<1)$ and computed the normal direction (derivative of $F)$, a line passing through the cells can be defined and assumed to be the fluid front.

The front motion is governed by the following equation:

$\frac{\partial F}{\partial t}+\vec{v} \cdot \nabla F=0$

Then, it is necessary to set the boundary condition. As the front is defined through the mesh, this technique use a layer of fictitious cells surrounding the front, in which the dependent variables
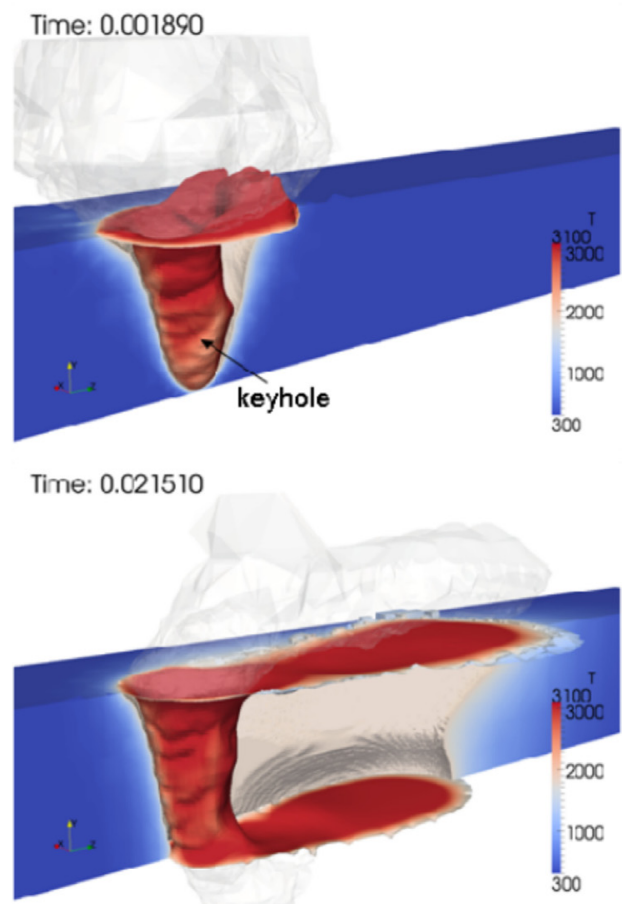

Fig. 11. Example of laser welding simulation with VOF method [54]. 

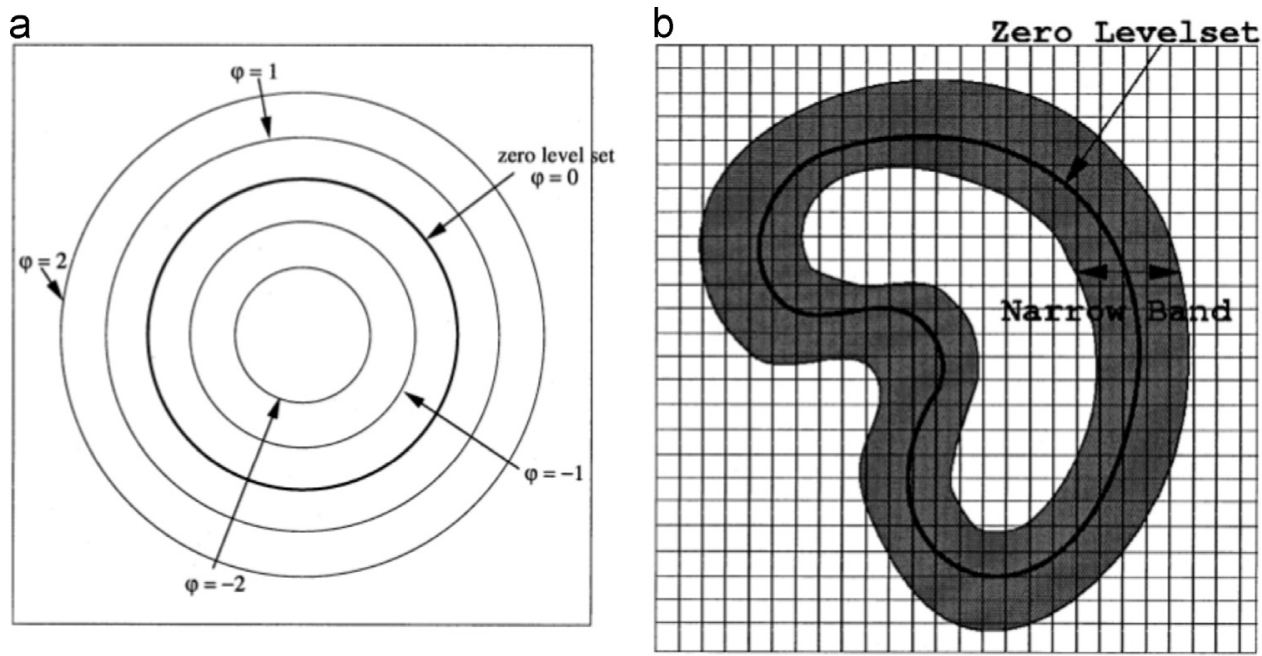

Fig. 12. Scheme of the Level Set principle: (a) level set function and (b) narrow band LS [36].

(velocity and pressure) are set (sleep, no-sleep, pressure conditions,...).

Applied to the simulation of laser welding, this technique have been wildly used by Otto et al. ([25,54,52] or [53]). Indeed, coupled to the finite volume discretization (OpenFoam) the VOF method is very efficient in terms of numerical costs. This make the algorithm particularly suitable to large simulations (Fig. 11).

Fig. 11, shows a result of transient laser welding. Looking at the ripples in front of the capillary, the capability of this tracking method to simulated the keyhole boundary is obvious.

\subsubsection{Level Set (LS) method}

The Level Set method is also an Eulerian free surface tracking method. This means that here again the free boundary is computed through the fix grid [51].

As shown in Fig. 12a, the LS variable $(\varphi)$ corresponds to the actual distance from the zero level set. Zero is the coordinates of the front. A narrow band method can also be used (Fig. 12b) in order to reduce the size of the problem (matrix) and so the computational cost [36].

As the coupling with the fluid flow is the same as previously, the partial differential equation solved to obtain the boundary motion is very similar to (26):

$\frac{\partial \varphi}{\partial t}+\vec{v} \cdot \nabla \varphi=0$

It should be noted that Eq. (27) is the simplest form of this convection (advection) equation. In the literature one can find more complex forms including terms for stabilization, control of the band thickness or surface tension [50].

Here the method does not give tools to implement boundary condition. That is why, in the case of laser welding simulation (or other multi-physical simulation), the constitutive equations have to be slightly adapted. As the fluid limit is inside the mesh grid, the condition equations are extended in the "boundary thickness" which means, in the domain:

- The heat source coming from the laser $(q)$ and the latent heat (Lvap) are set directly in the heat equation:

$$
\rho c_{p}^{e q} \dot{T}+\rho c_{p} \vec{v} \cdot \overrightarrow{\nabla T}=\vec{\nabla} \cdot \lambda \overrightarrow{\nabla T}+\left(q-m_{\text {vap }} L_{\text {vap }}\right) \delta(\phi)
$$

with $m_{\text {vap }}$ the evaporation rate.

- The surface tension $(\gamma)$ set directly in the momentum conservation equation:

$$
\begin{aligned}
\rho\left(\frac{\partial \vec{v}}{\partial t}+\vec{v} \cdot(\vec{\nabla} \cdot \vec{v})\right)= & \vec{\nabla} \cdot\left[-p I+\mu\left(\vec{\nabla} \vec{v}+\vec{\nabla} \vec{v}^{T}\right)\right]+\rho \vec{g} \\
& +F_{v o l}+(\gamma \kappa \vec{n}) \delta(\phi)
\end{aligned}
$$

The recoil pressure is usually taken into account through the evaporated flow rate as a "jump function" in the mass conservation [18] and in the Level Set equation [65]).

The results of simulation made with this technique are very interesting:

Fig. 13 shows capillary shapes obtained in two references. Similarly to the VOF method, the boundary description is quite efficient (ripples observed on the keyhole front).

It should be noted that introducing the surface tension (and pressure) as shown in Eq. (29) has to be treated very carefully. Indeed, as the free surface (liquid/vapor) phenomena modeling is the key to a successful simulation of welding, if the numerical errors during the discretization of this interface is not mastered, the numerical results could be quite far to the reality. Therefore, while they are very useful, the use commercial software (Comsol Multiphysics, Fluent, ...) needs a good understanding of the numerical method pre-implemented. Some quite recent numerical techniques can be used to enhance the simulation of the boundary gap: the "sharp interface method" [35] or the "balanced forced method" [24]. An application to laser welding simulation can be found in [55] focused on the surface tension treatment.

\subsubsection{Conclusion}

In the previous sections, related to "multi-physical" simulation, a summary of physical phenomena which have to be treated is made. Some crucial numerical points, as the simulation of the free boundary, are also presented. (An advantage of Eulerian methods is that the simulation can compute the keyhole collapsing and help to predicate defect formation such as porosity [56,44].) In conclusion, current best simulations take into account the multiple reflections of the laser, the heat transfers with liquid and gas flows, and the liquid/gas free boundary displacement. They also have taken the recoil pressure calculation very carefully. Indeed, the keyhole temperature and shape calculation is a key point in laser welding simulation.

Regardless of the numerical method, finite elements or finite volumes, it was observed that the most complete simulations were closer to the reality. Nevertheless, as these models are quite large, the calculations are still numerical performances (weeks or month 
a
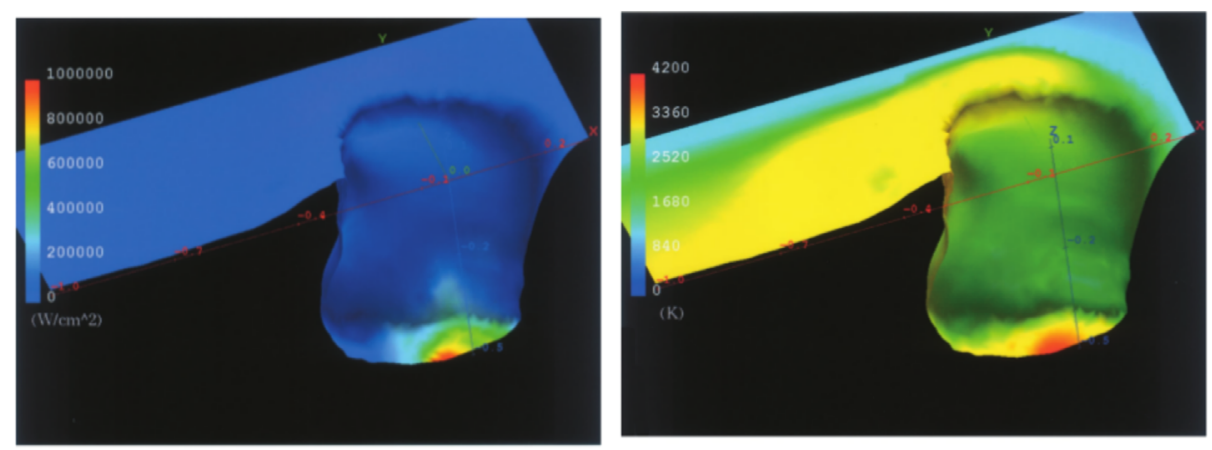

b
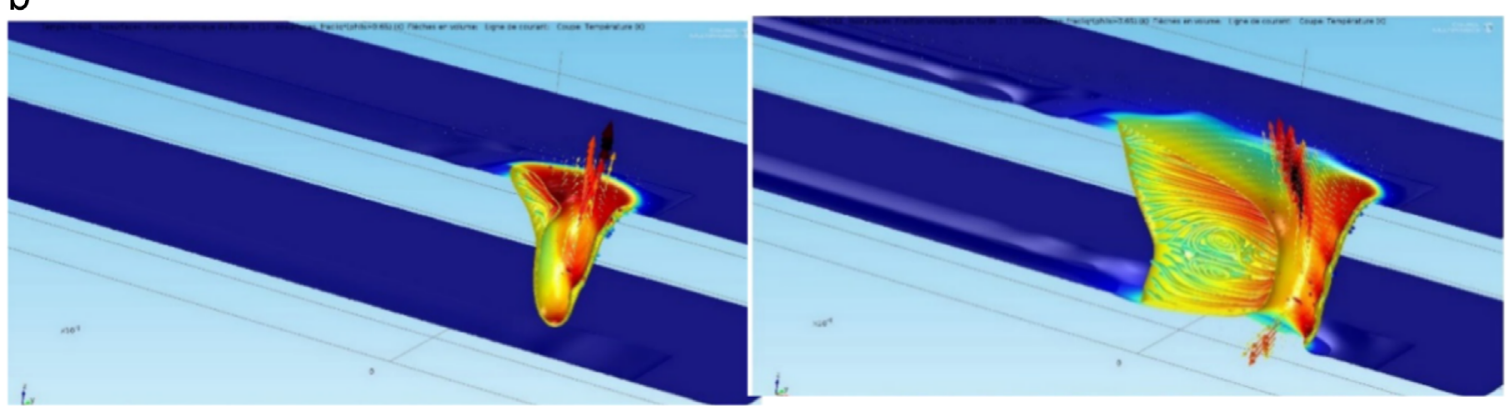

Fig. 13. Example of simulation results obtained with LS method: (a) [36] and (b) [16].

calculation times). That is why the most economical methods are preferred.

While they are recurrent in literature, a difficulty with Level Set and VOF methods is the mesh size needed to capture the small spatters occurring in laser welding. The use of "front tracking" methods, with a Lagrangian treatment of the interface and adapted to this effect [71] or using the most recent Moment of Fluid (Eulerian) method [20] the numerical size of the problem should be smaller and the simulation results could be better.

It has also been observed that very few experimental validations are made. Usually, only fusion zone shapes are checked. Control of velocity, temperature or keyhole shape is missing.

\section{Summary and conclusion}

This paper is a review of the different ways, shown in the literature, to simulate laser welding.

After having explained shortly the principle and the origin of laser, two modeling methodologies have been shown. Indeed, due to the high multi-physical aspect of laser welding simulation, phenomena are usually segregated.

The first is the thermo-mechanical simulation made to compute the residual strains and distortions due to this laser processing. These mechanical models consider a simplified thermal problem, but they can take into account the metallurgical transformations during the heating and cooling. Thus, it should be known that the thermal field is computed with some approximations, mainly at high temperature (near the fusion zone) where the model is the poorest. As the thermal transfer phenomena are approximated neglecting the fluid flow, the size of the numerical problem is reduced and these models can be used to large structure calculation.

The second is the multi-physical simulation. This last aims to compute a realistic thermal and velocity field in order to obtain with accuracy the fusion zone shape (weld bead). To do this, authors consider the laser beam multiple reflections, the vaporization creating the keyhole and the hydro-dynamics flows of the liquid and vapor. The liquid-vapor boundary is the seat of heat deposition and vaporization. Its numerical simulation is critical to obtain satisfactory results of the welding simulation. In this paper, three methods with their advantages and drawbacks have been shown.

Of course both methodologies are currently highly studied. The future works should be, for example, the enhancement of the material properties knowledge for the thermo-mechanical simulation. The simulations of very large and complete structures are also currently highly studied by using reduction model techniques for example.

For the multi-physical case, it is the thermo-physical properties at high temperature (liquid and vapor) that are missing. Moreover, the simulation of the gas behavior in the keyhole is also a current active research field.

Finally, considering the increase of computing resources, the unification thermo-mechanical and multi-physical simulation should also appear in future years.

\section{References}

[1] I. Alexeev, K.H. Leitz, A. Otto, M. Schmidt, Application of bessel beams for ultrafast laser volume structuring of non transparent media, Phys. Procedia 5 (2010) 533-540.

[2] E.H. Amara, R. Fabbro, Modelling of gas jet effect on the melt pool movements during deep penetration laser welding, J. Phys. D: Appl. Phys. 41 (2008).

[3] A. Anca, A. Cardona, J. Risso, V.D. Fachinotti, Finite element modeling of welding processes, Appl. Math. Modell. 35 (2011) 688-707.

[4] S.I. Anisimov, Vaporization of metal absorbing laser radiation, Sov. Phys. JETP 27 (1) (1968) 339-342.

[5] C. Bonacina, G. Comini, A. Fassano, M. Primicerio, Numerical solution of phasechange problems, Int. J. Heat Mass Transf. 16 (1973) 1825-1832.

[6] A.D. Brent, V.R. Voller, K.J. Reid, The enthalpy-porosity technique for modeling convection-diffusion phase change: application to the melting of pure metal, Numer. Heat Transf. 13 (3) (1988) 297-318.

[7] P.C. Carman, Fluid flow through granular beds, Trans. Inst. Chem. Eng. 75 (1997) 32-48, Jubilee Supplement.

[8] N. Chakraborty, The effects of turbulence on molten pool transport during melting and solidification processes in continuous conduction mode laser 
welding of copper-nickel dissimilar couple, Applied Thermal Engineering 29 (2009) 3618-3631.

[9] N. Chakraborty, S. Chakraborty, Modelling of turbulent molten pool convection in laser welding of a copper-nickel dissimilar couple, Int. J. Heat Mass Transf. 50 (2007) 1805-1822.

[10] X. Chen, H.-X. Wang, A calculation model for the evaporation recoil pressure in laser material processing, J. Phys. D: Appl. Phys. 34 (2001) 2637-2642.

[11] J.-H. Cho, S.-J. Na, Mechanism of keyhole formation and stability in stationary laser welding, J. Phys. D: Appl. Phys. 35 (2002) 1570-1576.

[12] J.-H. Cho, S.-J. Na, Implementation of real-time multiple reflection and fresnel absorption of laser beam in keyhole, J. Phys. D: Appl. Phys. 39 (2006) 5372-5378.

[13] W.-I. Cho, S.-J. Na, C. Thomy, F. Vollertsen, Numerical simulation of molten pool dynamics in high power disk laser welding, J. Mater. Process. Technol. 212 (2012) 262-275.

[14] J. Rahman Chukkan, M. Vasudevan, S. Muthukumaran, R. Ravi Kumar N. Chandrasekhar, Simulation of laser butt welding of \{AISI\} 3161 stainless steel sheet using various heat sources and experimental validation, J. Mater. Process. Technol. 219 (2015) 48-59.

[15] K. Contag, M. Karszewski, C. Stewen, A. Giesen, H. Hugel, Theoretical modelling and experimental investigations of the diode-pumped thin-disk yb:Yag laser, Quantum Electron. 29 (8) (1999) 697.

[16] M. Courtois, M. Carin, P. le Masson, S. Gaied, M. Balabane, A new approach to compute multi-reflections of laser beam in a keyhole for heat transfer and fluid flow modelling in laser welding, J. Phys. D: Appl. Phys. 46 (2013) 14.

[17] M. Courtois, M. Carin, P. le Masson, S. Gaied, M. Balabane, Modélisation thermo-hydraulique tridimensionnelle du soudage laser et validation expérimentale, Matér. Tech. 102 (404) (2014) 5.

[18] A.K. Dasgupta, J. Mazumder, P. Li, Physics of zinc vaporization and plasma absorption during $\mathrm{CO}_{2}$ laser welding, J. Appl. Phys. 102 (2007) 053108-1-15.

[19] F.M. Dickey, Laser Beam Shaping: Theory and Techniques, CRC Press, 2014.

[20] V. Dyadechko, M. Shashkov, Moment-of-Fluid Interface Reconstruction, Technical Report LA-UR-05-7571, Los Alamos National Laboratory, 2005.

[21] F. Faurea, J.-M. Bergheau, J.-B. Leblond, B. Souloumiac, New methods for numerical simulation of welding of large thin structures, Rev. Eur. Élém. 13 (3-4) (2004) 289-311.

[22] P. Ferro, H. Porzner, A. Tiziani, F. Bonollo, The influence of phase transformations on residual stresses induced by the welding process-3d and $2 \mathrm{~d}$ numerical models, Modell. Simul. Mater. Sci. Eng. 14 (2006) 117-136.

[23] R. Fortunier, J-M. Bergheau, Modelling of heat transfers, phase changes and mechanical behaviour during welding, Rev. Eur. Élém. 13:3(4) (2004) 231-245.

[24] M.M. Francois, S.J. Cummins, E.D. Dendy, D.B. Kothe, J.M. Sicilian, M. W. Williams, A balanced-force algorithm for continuous and sharp interfacial surface tension models within a volume tracking framework, J. Comput. Phys. 213 (1) (2006) 141-173.

[25] M. Geiger, K.H. Leitz, H. Koch, A. Otto, A 3d transient model of keyhole and melt pool dynamics in laser beam welding applied to the joining of zinc coated sheets, Prod. Eng. Res. Dev. 3 (2009) 127-136.

[26] J. Goldak, A. Chakravarti, M. Bibby, A new finite element model for welding heat sources, Metallurg. Trans. B 15B (1984) 299-305.

[27] E. le Guen, R. Fabbro, M. Carin, F. Coste, P. le Masson, Analysis of hybrid nd:yag laser-mag arc welding processes, Opt. Laser Technol. 43 (7) (2011) 1155-1166.

[28] H. Haken, Light: Laser Light Dynamics, North-Holland Publishing Company, 1985.

[29] S-W. Han, J. Ahn, S-J. Na, A Study on Ray Tracing Method for cfd Simulations of Laser Keyhole Welding: Progressive Search Method, International Institute of Welding, pages Doc. 212-1379-15, 2015.

[30] K. Hirano, R. Fabbro, Experimental investigation of hydrodynamics of melt layer during laser cutting of steel, J. Phys. D: Appl. Phys. 44 (10) (2011) 105502.

[31] K. Hirano, R. Fabbro, M. Muller, Experimental determination of temperature threshold for melt surface deformation during laser interaction on iron at atmospheric pressure, J. Phys. D: Appl. Phys. 44 (43) (2004) 17.

[32] C.W. Hirt, A.A. Amsden, J.L. Cook, An arbitrary Lagrangian-Eulerian computing method for all flow speeds, J. Comput. Phys. 135 (1997) 203-216.

[33] C.W. Hirt, B.D. Nichols, Volume of fluid (vof) method for the dynamics of free boundaries, J. Comput. Phys. 39 (1981) 201-225.

[34] C.B. Hitz, J.J. Ewing, J. Hecht, Introduction to Laser Technology, John Wiley \& Sons, 2012.

[35] M. Kang, R.P. Fedkiw, X.D. Liu, A boundary condition capturing method for multiphase incompressible flow, J. Sci. Comput. 15 (3) (2000) 323-360.

[36] H. Ki, P.S. Mohanty, J. Mazumder, Modeling of laser keyhole welding. Part I. mathematical modeling, numerical methodology, role of recoil pressure, multiple reflections, and free surface evolution, Metall. Mater. Tans. A 33A (2002) 1817-1830.

[37] C.J. Knight, Theoretical modeling of rapid surface vaporization with back pressure, AIAA J. 17 (5) (1979).

[38] D.P. Koistinen, R.E. Marburger, A general equation prescribing the extent of the austenite-martensite transformation in pure iron-carbon alloys and plain carbon steels, Acta Metall. 7 (1) (1959) 59-60.

[39] S. Kumar, R. Awasthi, C.S. Viswanadham, K. Bhanumurthy, G.K. Dey, Residual stresses in laser welded. ASTM A387 Grade 91 steel, Mater. Sci. Eng. A575 (2013) 160-168.

[40] S. Kumar, R. Awasthi, C.S. Viswanadham, K. Bhanumurthy, G.K. Dey, Modelling of heat transfers, phase changes and mechanical behaviour during welding, Thermo-metallurgical and Thermo-mechanical Computations for Laser Welded Joint in $9 \mathrm{Cr}-1 \mathrm{Mo}(\mathrm{V}, \mathrm{Nb})$ ferritic/martensitic steel, vol. 59, 2014, pp. 211220.

[41] G. Labeas, I. Diamantakos, Laser beam welding residual stresses of cracked t-joints, Theor. Appl. Fract. Mech. 63-64 (2013) 69-76.

[42] J. Leblond, J. Devaux, A new kinetic model for anisothermal metallurgical transformations in steels including effects of austenite grain size, Acta Metall. 32 (1984) 137-146.

[43] X. Li, L. Wang, L. Yang, J. Wang, K. Li, Modeling of temperature field and pool formation during linear laser welding of dp1000 steel, J. Mater. Process. Technol. 214 (2014) 1844-1851.

[44] F. Lu, X. Li, Z. Li, X. Tang, H. Cui, Formation and influence mechanism of keyhole-induced porosity in deep-penetration laser welding based on $3 \mathrm{~d}$ transient modeling, Int. J. Heat Mass Transf. 90 (2015) 1143-1152.

[45] P. Martinson, S. Daneshpour, M. Kocak, S. Riekehr, P. Staron, Residual stress analysis of laser spot welding of steel sheets, Mater. Des. 30 (9) (2009) 3351-3359.

[46] A. Matsunawa, V. Semak, The simulation of front keyhole wall dynamics during laser welding, J. Phys. D: Appl. Phys. 30 (1997) 798-809.

[47] M. Medale, S. Rabier, C. Xhaard, A thermo-hydraulic numerical model for high energy welding processes, Rev. Eur. Élém. 13 (3-4) (2004) 207-229.

[48] M. Medale, C. Touvrey, R. Fabbro, An axi-symmetric thermo-hydraulic model to better understand spot laser welding, Eur. J. Comput. Mech. 17 (5-7) (2008) $795-806$.

[49] M. Medale, C. Xhaard, R. Fabbro, A thermo-hydraulic numerical model to study spot laser welding, C. R. Mec. (2007) 7.

[50] S. Osher, R. Fedkiw, Level Set Methods and Dynamic Implicit Surfaces, Springer Science, Business Media, 2006.

[51] S. Osher, J.A. Sethian, Fronts propagating with curvature dependent speed: algorithms based on Hamilton-Jacobi formulations, J. Comput. Phys. 79 (1988) $12-49$.

[52] A. Otto, H. Koch, K.-H. Leitz, M. Schmidt, Numerical simulations-a versatile approach for better understanding dynamics in laser material processing, Phys. Procedia 12 (2011) 11-20.

[53] A. Otto, H. Koch, R.G. Vazquez, Multiphysical simulation of laser material processing, Phys. Procedia 39 (2012) 843-852.

[54] A. Otto, M. Schmidt, Towards a universal numerical simulation model for laser material processing, Phys. Procedia 5 (2010) 35-46.

[55] S. Pang, L. Chen, J. Zhou, Y. Yin, T. Chen, A three-dimensional sharp interface model for self-consistent keyhole and weld pool dynamics in deep penetration laser welding, J. Phys. D: Appl. Phys. 44 (2) (2011) 025301.

[56] S. Pang, W. Chen, W. Wang, A quantitative model of keyhole instability induced porosity in laser welding of titanium alloy, Metall. Mater. Trans. A 45 (6) (2014) 2808-2818.

[57] S. Pang, X. Chen, J. Zhou, X. Shao, C. Wang, 3d transient multiphase model for keyhole, vapor plume, and weld pool dynamics in laser welding including the ambient pressure effect, Opt. Lasers Eng. 74 (2015) 47-58.

[58] S. Pang, K. Hirano, R. Fabbro, T. Jiang, Explanation of penetration depth variation during laser welding under variable ambient pressure, J. Las. Appl. 27 (2) (2015) 022007.

[59] J. Peatross, M. Ware, Physics of Light and Optics: Black and White Edition, BYU Bookstore, 2007.

[60] K.R. Renk, Basics of Laser Physics, Springer, 2012.

[61] P. Sahoo, T. DebRoy, M.J. McNallan, Surface tension of binary metal-surface active solute systems under conditions relevant to welding metallurgy, Metall. Trans. B 19 (1988) 483-491.

[62] C. Seang, A.K. David, E. Ragneau, Nd:yag laser welding of sheet metal assembly: transformation induced volume strain affect on elastoplastic model Phys. Procedia 41 (2013) 448-459.

[63] A.E. Siegman, Lasers, University Science Books, 1986.

[64] O. Svelto, Principles of Lasers, Springer, 2000.

[65] W. Tan, N.S. Bailey, Y.C. Shin, Investigation of keyhole plume and molten poo based on a three-dimensional dynamic model with sharpinterface formulation, J. Phys. D: Appl. Phys. 46 (2013) 12.

[66] W. Tan, Y.C. Shin, Analysis of multi-phase interaction and its effects on keyhole dynamics with a multi-physics numerical model, J. Phys. D: Appl. Phys. 47 (2014) 17.

[67] G. Tirand, C. Arvieu, E. Lacoste, J.-M. Quenisset, Control of aluminium laser welding conditions with the help of numerical modelling, J. Mater. Process. Technol. 213 (3) (2013) 337-348.

[68] F. Trager, Springer Handbook of Lasers and Optics, Springer Science, Business Media, 2007.

[69] M. Zain ul Abdein, D. Nelias, J.-F. Jullien, D. Deloison, Prediction of laser beam welding-induced distortions and residual stresses by numerical simulation for aeronautic application, J. Mater. Process. Technol. 209 (6) (2009) 2907-2917.

[70] M. Zain ul Abdein, D. Nelias, J.-F. Jullien, D. Deloison, Experimental investigation and finite element simulation of laser beam welding induced residual stresses and distortions in thin sheets of $\{A A\}$ 6056-t4, Mater. Sci. Eng. A 527 (12) (2010) 3025-3039.

[71] S.O. Unverdiand, G. Tryggvason, A front-tracking method for viscous, incompressible, multi-fluid flows, J. Comput. Phys. 100 (1) (1992) 25-37.

[72] M.J. Weber, Handbook of Lasers, CRC Press LLC, 2001. 